\title{
Dermatoscopia
}

\section{ACHADOS DERMATOSCÓPICOS NO LÍQUEN ESTRIADO}

\author{
Carlos Gustavo Carneiro de Castro', Dayana Monteiro Coutinho², Aline Serruya ${ }^{3}$, Fernanda Lourenço Prestes ${ }^{4}$, Lara \\ Braga $^{3}$, Glaura Tinoco Plata ${ }^{7}$ \\ 'Sócio efetivo da Sociedade Brasileira de Dermatologia/Dermatologist certified by the Brazilian Society of \\ Dermatology \\ ${ }^{2}$ Aluna/Medical Student, Universidade Federal de Juiz de Fora, Brasil \\ ${ }^{3}$ Aluna do $6^{\circ}$ ano de Medicina do FTESM/Medical Student of FTESM, Brasil \\ ${ }^{4}$ Graduada em Medicina/Medical Graduated, UNESA. Pós Graduação em Saúde da Família pelo UNASUS/UERJ, \\ Brasil
}

\begin{abstract}
RESUMO - O líquen estriado é uma dermatose papulosa de baixa prevalência, acometendo preferencialmente crianças, sendo mais frequente no sexo feminino. Clinicamente, evolvi com aparecimento abrupto de pápulas eritematovioláceas ou hipopigmentadas, geralmente não pruriginosas e unilaterais, com predileção para extremidades, seguindo as linhas de Blaschko. Relatamos um caso de líquen estriado com achados atípicos, no qual a dermatostopia, além do exame histopatológico, foram úteis na avaliação diagnóstica.
\end{abstract}

PALAVRAS-CHAVE - Dermoscopia; Doenças da pele; Dermatoses da mão.

\section{DERMOSCOPY FINDINGS IN LICHEN STRIATUS}

ABSTRACT - Lichen striatus is a papulous dermatosis with low prevalence, affecting mainly children and it is more frequent on the female population. Clinically, it evolves with abrupt onset with erythematous violaceous or hypopigmented papules, generally with non-pruritic and with unilateral distribution, with predilection for the extremities, following the Bhlaschko lines. We report a case of lichen striatus with atypical features and in this case, in addition to the histopathological exam, dermoscopy was useful on the diagnostic evaluation.

KEY WORDS - Dermoscopy; Hand dermatoses; Skin diseases.

Conflitos de interesse: Os autores declaram não possuir conflitos de interesse.

No conflicts of interest.

Suporte financeiro: O presente trabalho não foi suportado por nenhum subsídio ou bolsa.

No sponsorship or scholarship granted.

Recebido/Received - Novembro/November 2014; Aceite/Accepted - Dezembro/December 2014

Correspondência:

Dr. Carlos Gustavo Carneiro de Castro

Rua Voluntários da Pátria 445, sala 702

Botafogo, Rio de Janeiro

RJ, Brasil, CEP 22270-000 


\section{Dermatoscopia}

\section{INTRODUÇÃO}

O líquen estriado, uma dermatose basicamente papulosa, autolimitada e rara, é uma condição assintomática que ocorre mais comumente em crianças em adolescentes, podendo ocasionalmente ocorrer em adultos'. Acomete mais frequentemente mulheres, na proporção de 2:12. Sua etiologia é desconhecida, mas há autores que consideram a doença uma manifestação do mosaicismo, onde o sistema imune passa a atacar células epiteliais alteradas geneticamente em distribuição linear ${ }^{1}$. Atopia e fatores ambientais, como infecções, podem estar associados ao surgimento do líquen estriado ${ }^{2}$. Sua apresentação consiste em pápulas, por vezes descamativas, que podem ser achatadas, de surgimento súbito, normalmente não pruriginosas, usualmente unilateral, acometendo principalmente extremidades, raramente tronco e face ${ }^{3}$. Pode haver, também, acometimento ungueal. As lesões tipicamente seguem as linhas de Blaschko ${ }^{3}$. Por vezes as lesões regridem espontaneamente dentro de um ano. $O$ tratamento consiste em corticóide tópico ou inibidores de calcineurina ou imiquimode ${ }^{2}$. À histopatologia é possível observar reação liquenóide com vacuolização da camada basal e inflamação de anexos ${ }^{3}$. Na dermatoscopia pode-se observar características vasculares e não-vasculares ${ }^{4}$. Estruturas esbranquiçadas com limites bem definidos podem ser observadas em lesões papulosas tendo um aspecto semelhante ao das estrias de Wickham no líquen plano. Pode-se observar ainda uma despigmentação simulando uma cicatriz esbranquiçada recentemente descrita na ceratose liquenóide-líquen plano símile ${ }^{4}$. Em lesões psoriasiformes distais pode-se observar características dermatoscópicas como: estruturas ceratósicas acastanhadas a amareladas, estruturas amarelas e estruturas com aspecto "de noz-moscada" com pontos vermelhos em seu interior ${ }^{4}$.

É importante diferenciar o líquen estriado de outras doenças que também cursam com lesões que acompanham as linhas de Blaschko, entre elas a doença do enxerto versus hospedeiro linear, psoríase linear e o líquen plano, seu principal diagnóstico diferencial ${ }^{1}$.

Relatamos um caso clínico de líquen estriado iniciando na adolescência e de localização infrequente no qual a dermatoscopia auxiliou na avaliação diagnóstica.

\section{RELATO DE CASO}

Paciente masculino, 17 anos, notou o surgimento há aproximadamente 14 meses, de pápulas achatadas hipercrômicas assintomáticas no flanco direito e dorso seguindo as linhas de Blascko. Foi realizada biópsia excisional de uma das pápulas e o exame histopatológica da lesão foi compatível com líquen estriado. Foi iniciado tratamento com tacrolimus $0,1 \%$ pomada, duas vezes ao dia, com melhora importante das lesões em 4 semanas. Na história patológica pregressa apresenta vitiligo na região pubiana e asma. Nega doenças autoimunes e doenças dermatológicas na família. Ao exame dermatológico apresenta pápulas hipercrômicas em grande número em sua maioria com tamanho variando entre 2 a $5 \mathrm{~mm}$ e máculas hiperpigmentadas seguindo o trajeto das linhas de Blascko. Ao exame dermatoscópico de uma lesão, foram visualizados pontos enegrecidos com distribuição salpicada no seu interior e bem como áreas esbranquiçadas. $O$ exame histopatológico corado pela hematoxilina e eosina demonstrou epiderme com hiperceratose, vacuolização da camada basal e derme exibindo infiltrado inflamatório linfocitário em faixa na porção papilar, perivascular e perianexial. Melanófogos presentes.

\section{DISCUSSÃO}

O líquen estriado, também chamado de dermatose linear liquenóide, surge mais comumente na infância e é discretamente mais prevalente em pacientes do sexo feminino. Manifesta-se clinicamente por pápulas hipopigmentadas ou eritematovioláceas (Fig. 1). São lesões de superfície lisa, que variam de 2 a $4 \mathrm{~mm}$ de tamanho e que coalescem em disposição linear, formando uma faixa descontínua, seguindo as linhas de

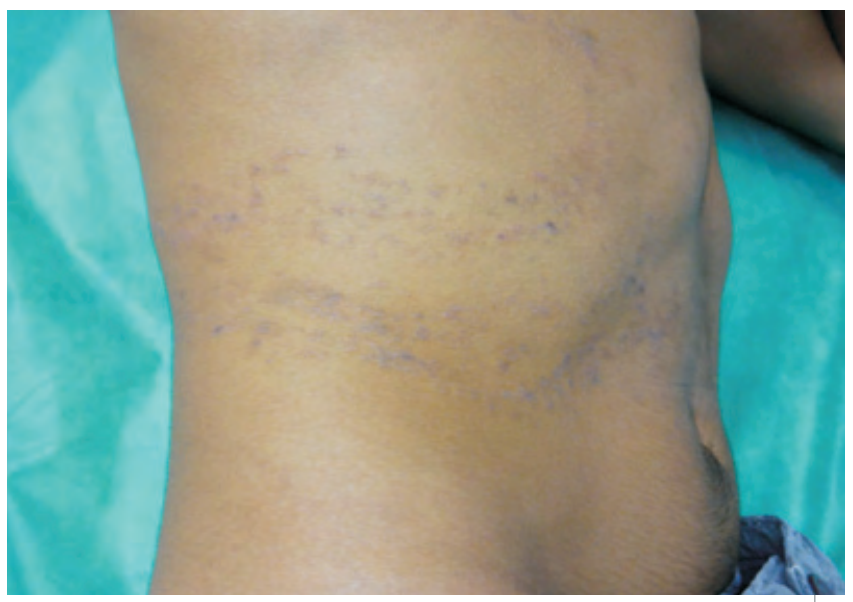

Fig 1 - Líquen estriado seguindo as linhas de Blascko. 


\section{Dermatoscopia}

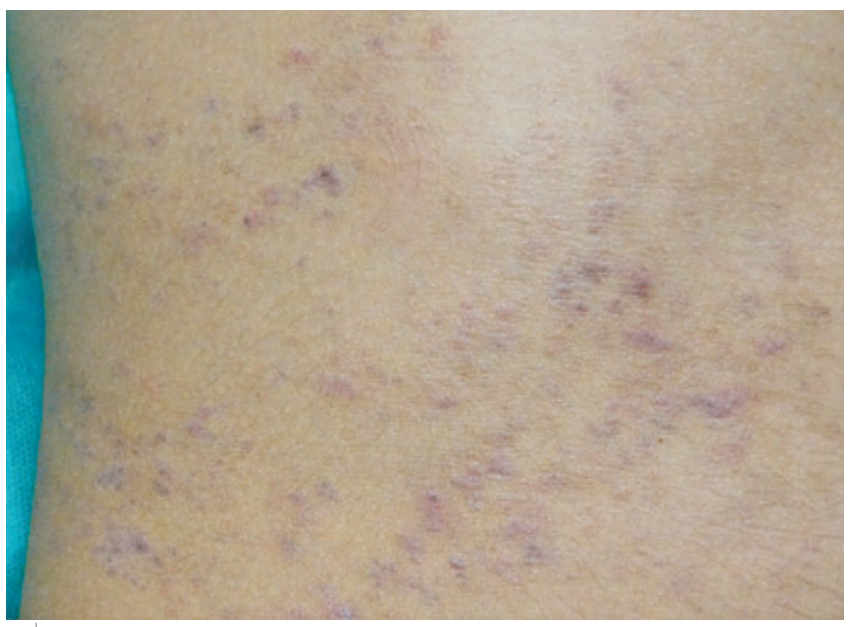

Fig 2 - Pápulas eritematovioláceas no tronco.

Blaschko (Fig. 2). Devido a esta apresentação sugere-se a possibilidade de participação de nervos e vasos linfáticos. Normalmente são unilaterais, assintomáticas e frequentemente localizam-se nas extremidades dos membros. No presente caso relatado, foram descritas pápulas hipercrômicas e achatadas em flanco direito e dorso, de início na adolescência em paciente do sexo

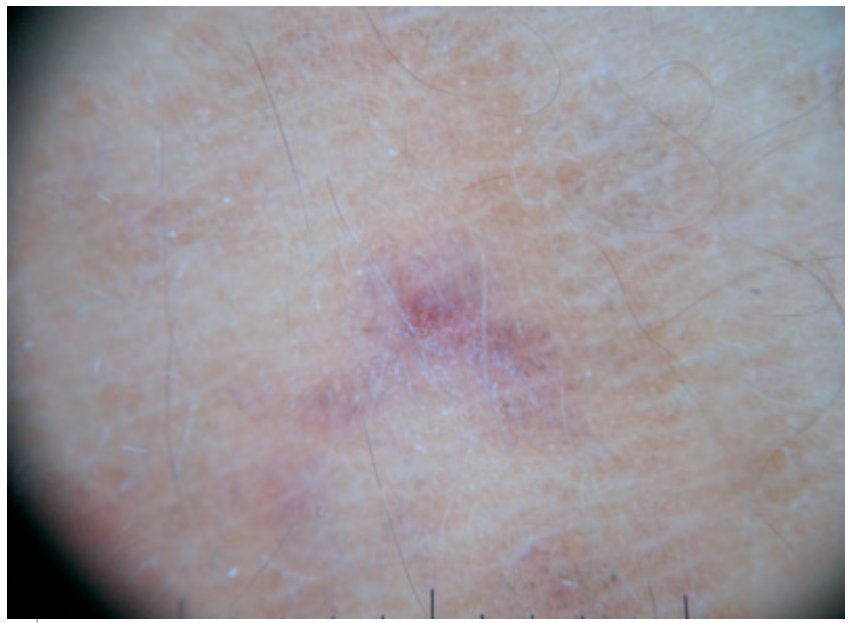

Fig 3 - Dermatoscopia demonstrando pontos enegrecidos e áreas esbranquiçadas na lesão de líquen estriado em involução.

masculino ${ }^{5}$ Tal apresentação clínica foi atípica no que diz respeito a topografia das lesões, idade de início e o sexo do paciente. De início abrupto, porém auto limitado, a dermatose liquenóide pode ter relação com

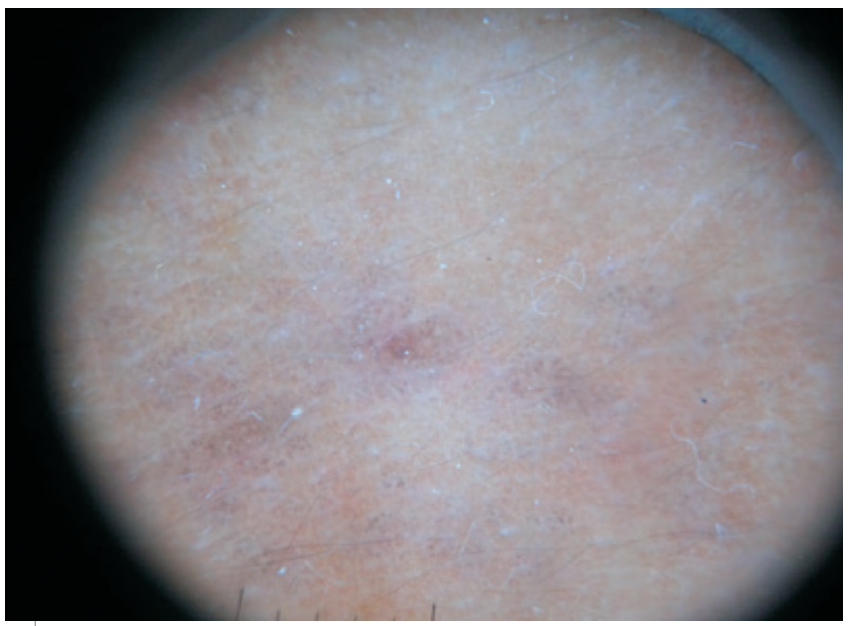

Fig 4 - Maior detalhe dos achados dermatoscópicos.

vacinas como BCG e Hepatite $B$; infecções como a varicela; traumatismos; medicamentos (adalimumabe, metronidazol) e gravidez. Associação com dermatite atópica rinite alérgica e asma também já foram descri$\operatorname{tas}^{6}$ e, no caso clínico apresentado foi encontrada história de asma corroborando esta relação possível com atopia. Foi encontrado ainda, no paciente em questão a história de vitiligo que ocorre comumente em associação a outras doenças com provável participação de autoimunidade na patogênese como parece ser o caso do líquen estriado.

Eventualmente podem ocorrer alterações ungueais, como estriações longitudinais, adelgaçamento da lâmina ungueal e onicosquizia. Além disso, o líquen estriado pode ter extensão do infiltrado à derme profunda, envolvendo vasos, folículos pilosos e glândulas sudoríparas. Na histopatologia demonstra infiltrado linfo-histiocitário focal e liquenóide, com envolvimento perianexial superficial e profundo ${ }^{7}$. Pode-se encontrar ainda na histopatologia degenaração vacuolar da camada basal, necrose múltipla de ceratinócitos e queda de pigmento melânico' . A liquefação da camada basal seguida da queda de melanina na derme é uma provável explicação para o achado dermatoscópico de pontos enegrecidos com aspecto salpicado observados nas lesões do paciente do caso apresentando. Outros achados visualizados ao exame dermatoscópio foram áreas esbranquiçadas as quais são encontradas em lesões em regressão como no caso do nosso paciente ${ }^{4}$.

O líquen plano assim como outras dermatoses inflamatórias adquiridas que seguem as linhas de Blaschko, como a erupção liquenóide a droga, doença enxerto-versus-hospedeiro crônico liquenóide, lúpus 


\section{Dermatoscopia}

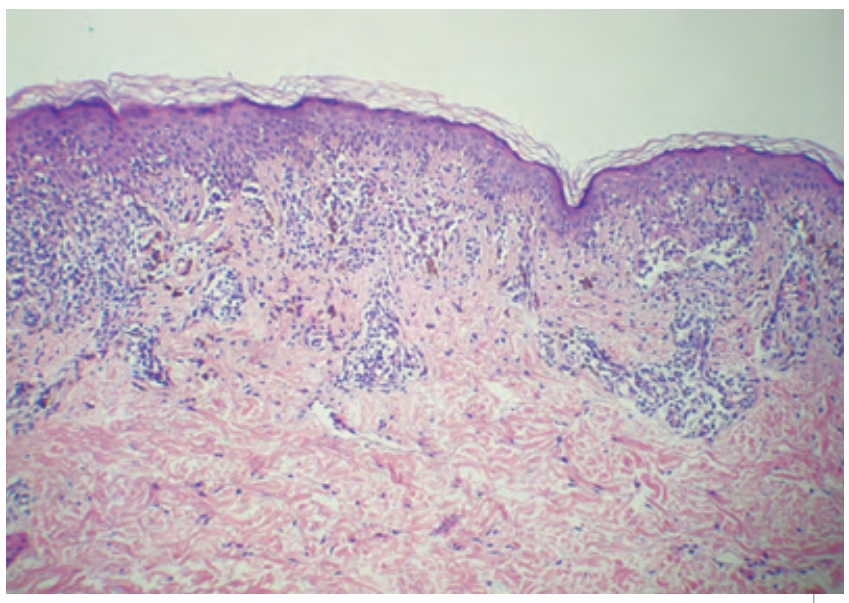

Fig 5 - Hiperceratose, acantose irregular, infiltrado liquenóide mononuclear com borramento da interface dermo epidérmica $(H \& E, x 40)$.

eritematoso, psoríase entre outras, são importantes diagnósticos diferenciais. O líquen plano linear também segue as linhas de Blaschko, porem diferencia-se do estriado por ser pruriginoso, com lesões poligonais e apresentam estrias de Wickham ${ }^{1,8}$. No caso relatado, embora as lesões fossem violáceas e lineares, esses achados estavam ausentes. Devido ao fato de geralmente ocorrer involução espontânea das lesões num período aproximado de até 12 meses e aos raros casos de recidiva, a conduta expectante pode ser uma opção. Porém, o uso de corticóide tópico e inibidores da calcineurina como o tacrolimus estão indicados para a diminuição do processo evolutivo9. Foi iniciado então tratamento tópico com tacrolimus 0,1\% pomada, duas vezes ao dia, durante 4 semanas havendo uma melhora importante do quadro com diminuição do relevo e da dor das lesões, e aparecimento de hipercromia residual

\section{CONCLUSÃO}

O caso apresentado tem características atípicas de líquen estriado que apesar de ser uma dermatose infrequente pode causar um impacto negativo psicossocial importante numa faixa etária em que a personalidade do indivíduo está em formação. Estes aspectos motivaram a escolha terapêutica do inibidor da calcineurina no caso em questão, que apresenta menor risco de efeitos adversos na pele em comparação com corticóides.

Existe escassez de publicação na literatura sobre achados dermatoscópicos nesta dermatose. Os pontos enegrecidos descritos no presente trabalho podem

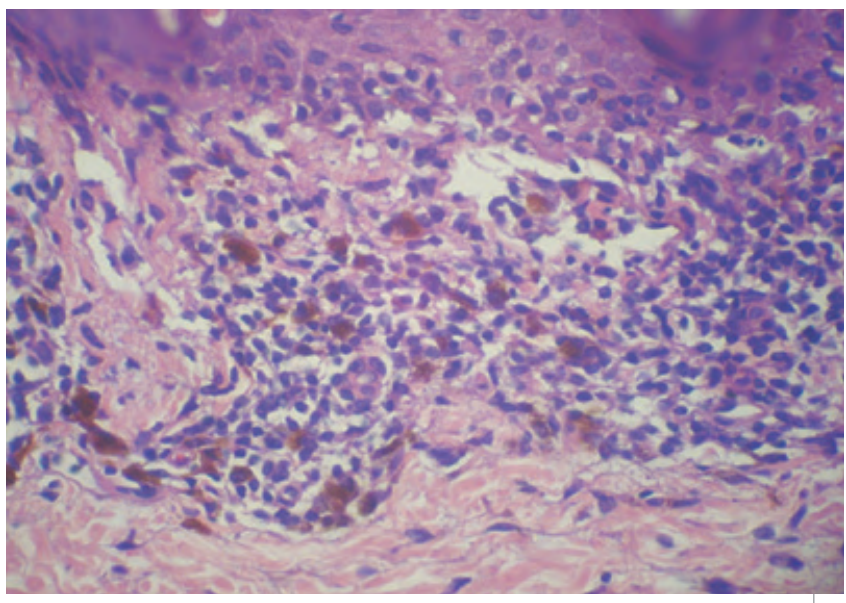

Fig 6 - Detalhe do borramento da interface dermo epidérmica por linfócitos, melanófagos e vacuolização da camada basal (H\&E, x100).

ajudar na diferenciação de outras dermatoses que acometem a linha de Bhlaschko e que portanto são contempladas no diagnóstico diferencial desta doença. A dermatoscopia facilita ainda a percepção de áreas esbranquiçadas que podem demonstrar a fase involutiva da doença, ajudando no acompanhamento da resposta terapêutica.

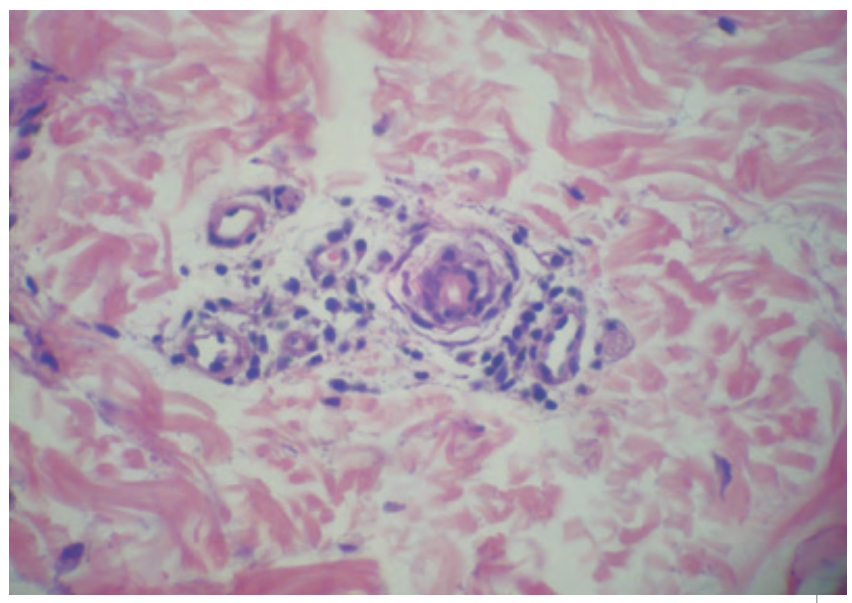

Fig 7 - Presença de infiltrado mononuclear na derme média ao redor de vénulas e estruturas anexiais ( $H \& E$, $\mathrm{x} 40$ ).

\section{REFERÊNCIAS}

1. Fogagnolo L, Barreto JA, Soares CT, Marinho FC, Nassif PW. Líquen estriado no adulto. An Bras Dermatol. $2011 ; 86(1): 142-5$. 


\section{Dermatoscopia}

2. Ramos C, Rodríguez S, Quijano E, Gutiérrez Z, Cortez-Franco F, Fuertes $C$, et al. Liquen estriado del adulto: experiencia en un servicio de Dermatología. Dermatol Peru. 2012; 22(1):16-20.

3. Kurokawa M, Kikuchi H, Ogata K, Setoyama M. Bilateral Lichen Striatus. J Dermatol. 2004; $31(2): 129-32$.

4. Segura P, Romero M, Gonzalvo P, Garcia S, Iglesias J, Juanes J. Lichen striatus in na adult folowing trauma with central nail plate involvement and its dermoscopy features. IntJ Dermatol. 2008, 47:324-5.

5. Shiohara T, Kano Y. Lichen planus and lichenoid dermatoses. In: Bolognia J, Jorizzo JL, Rapini RP, editors. Dermatology. $2^{\text {nd }}$ ed. Spain: Mosby Elsevier; 2008. p.159-80.
6. E. Tavares, JA Dores, J Aranha. Liquen estriado no adulto. Med. Cutan Iber Lat Am 2013; $41(4): 186-9$.

7. Mobini N, Toussaint S, Kamino H. Non Infectious erythematous papular and squamous diseases. In: Elder DE, Elenitsas R, Johnson Jr BL, Murphy GF, editors. Lever's Histopathology of the skin. $9^{\text {th }}$ ed. Philadelphia: Lippincott Williams \& Wilkins; 2005. p. 179-214.

8. Rubio FA, Robayna G, Herranz P, Lucas R, Hernandez-Cano N, Contreras $F$, et al. Linear lichen planus and lichen striatus: is there an intermediate form between these Conditions? Clin Exp Dermatol. 1997; 22:61-2.

9. Kim J. Lichen Striatus Yale: Yale Medical Center; 2010. 